\title{
Quality of life, self-stigma, and coping strategies in patients with neurotic spectrum disorders: a cross-sectional study
}

This article was published in the following Dove Medical Press journal: Psychology Research and Behavior Management

\author{
Michaela Holubova ${ }^{1-3}$ \\ Jan Prasko 1,2,4 \\ Marie Ociskova ${ }^{1,2}$ \\ Kryštof Kantor ${ }^{1,2}$ \\ Jakub Vanek ${ }^{1,2}$ \\ Milos Slepecky ${ }^{4}$ \\ Kristyna Vrbova ${ }^{1,2}$ \\ 'Department of Psychiatry, University \\ Hospital Olomouc, Olomouc, Czech \\ Republic; ${ }^{2}$ Faculty of Medicine \\ and Dentistry, Palacky University \\ Olomouc, Olomouc, Czech Republic: \\ ${ }^{3}$ Department of Psychiatry, Hospital \\ Liberec, Liberec, Czech Republic; \\ ${ }^{4}$ Department of Psychology Sciences, \\ Faculty of Social Science and Health \\ Care, Constantine the Philosopher \\ University in Nitra, Nitra, Slovakia
}

Correspondence: Jan Prasko Department of Psychiatry, University Hospital Olomouc, 6 Ulice IP Pavlova, Olomouc 775 20, Czech Republic Email praskojan@seznam.cz
Background: Modern psychiatry focuses on self-stigma, coping strategies, and quality of life (QoL). This study looked at relationships among severity of symptoms, self-stigma, demographics, coping strategies, and QoL in patients with neurotic spectrum disorders.

Methods: A total of 153 clinically stable participants who met criteria for generalized anxiety disorder, social phobia, panic disorder, agoraphobia, mixed anxiety-depressive disorder, adjustment disorders, somatoform disorders, or obsessive-compulsive disorder were included in a cross-sectional study. Psychiatrists examined patients during regular psychiatric checkups. Patients completed the Quality of Life Satisfaction and Enjoyment Questionnaire (Q-LES-Q), Internalized Stigma of Mental Illness Scale (ISMI), a sociodemographic questionnaire, the Stress Coping Style Questionnaire (Strategie Zvládání Stresu [SVF] 78), and the Clinical Global Impression (CGI) scale.

Results: The diagnostic subgroups differed significantly in age and use of negative coping strategies, but not in other measured clinical or psychological variables. The findings showed that neither sex nor partnership played a role in perceived QoL. All Q-LES-Q domains correlated negatively with all ISMI domains, except school/study. Unemployed and employed groups of patients differed in QoL. Each of the coping strategies, except the need for social support, was related to self-stigma. The findings showed that sex, partnership, education, and employment played no role in self-stigma. No differences between sexes in positive coping strategies, severity of disorder, self-stigma, or QoL were found. QoL correlated significantly with all coping strategies, except for guilt denial. Multiple regression showed the most important factors to be positive coping, employment, and overall self-stigma rating, explaining 32.9\% of QoL. Mediation analysis showed self-stigma level and negative coping strategies to be the most influential. The most substantial factors associated with self-stigma, as indicated by regression analysis, were Q-LES-Q total, subjective CGI, and positive coping strategies, which clarified $44.5 \%$ of the ISMI. Conclusion: The study confirmed associations among self-stigma, quality of life, disorder severity, and coping strategies of outpatients with neurotic spectrum disorders.

Keywords: self-stigma, quality of life, coping strategies, neurotic spectrum disorders

\section{Introduction}

ICD10 category F40-48, specifically "Neurotic disorders, stress-induced disorders, and somatoform disorders", covers a wide range of specific disorders, such as phobic anxiety disorders (social phobia), other anxiety disorders (generalized anxiety disorder [GAD], panic disorder), somatoform disorders, reaction to severe stress and adjustment disorders, obsessive-compulsive disorder (OCD), and dissociative disorders. ${ }^{1}$ For brevity, we talk about neurotic spectrum disorders (NsDs). NsDs are accompanied 
by high health-care utilization, significant impairment, and considerable economic burden. According to epidemiological surveys, a third of the population suffers from an NsD during their lifetime, with higher incidence in women and especially the middle-aged. ${ }^{2}$ They are the most common reason for the first visit to a psychiatrist or general practitioner for psychological problems in the Czech Republic. ${ }^{3}$ NsDs are the most prevalent psychiatric problems connected with a high burden of disease. ${ }^{1}$ Core manifestations of NsDs are tension, appraising situations as demanding, worries or ruminations, ineffective coping strategies, inability to control drives, a tendency to complain, and experiencing negative emotions, such as panic (immediate threat), anxiety (future threats), and depression (chronic stress). ${ }^{4}$

The main reason for assessing quality of life (QoL) in different types of NsD is that most studies describe reduced QoL in connection with neuroticism or anxiety., ${ }^{5,6}$ Also, clinical practice advocates that anxiety symptoms are negatively related to QoL. ${ }^{7,8}$ Specific subtypes of NsD may differ in significantly various QoL domains. ${ }^{9}$ However, there have not been many studies to measure differences in QoL in various subgroups of NsD or distinguish the effect of symptom severity on QoL. ${ }^{9}$ In Cramer et al, ${ }^{10}$ panic disorder, social phobia, and GAD had a negative effect on QoL, OCD had only a minor influence, and agoraphobia did not affect QoL at all.

According to Weidle et al, ${ }^{11}$ QoL increased while anxiety symptoms decreased in children with OCD. Youth with social anxiety reported lower QoL in association with higher severity of anxiety symptoms. ${ }^{12}$ Furthermore, lower socioeconomic status, ethnic minority, and female sex are described as predictors of reduced QoL and higher intensity of anxiety symptoms. ${ }^{13,14}$

While all NsDs seem to cause impairment through the distress connected with symptoms, avoidance, and selfstigma, there has been no description of which specific aspects of QoL are affected in different subtypes of NsD. ${ }^{15}$ For example, those with social anxiety disorder are more likely to have reduced QoL in the social domain of the World Health Organization Quality of Life - brief assessment, which includes personal relationships, social support, and social relationships. ${ }^{16}$

A standard definition of QoL has not been established. The World Health Organization outlines QoL as an individual's perception of their position in life in the context of the culture and value systems in which they live and in relation to their goals, expectations, standards, and concerns. ${ }^{17}$ QoL is related to such conditions as life-threatening illness and chronic and long-term disorders, which lead to a noticeable decrease in QoL. ${ }^{18,19}$ Every area of a patient's life affected by an illness, such as occupational and school functioning, household functioning, social functioning, emotional functioning, life satisfaction, and functional performance, may be included as a part of the multidimensional QoL model. ${ }^{20} \mathrm{QoL}$ assessment is based on a subjective observation of a patient's life. This approach was built on several sociological surveys that demonstrated that objective life circumstances (such as income or education) have only a marginal effect on QoL. ${ }^{21}$ Also, it is recommended to avoid the theoretical concepts and focus on personal observations associated with health. ${ }^{5}$

QoL could be connected to other psychological or social processes. One of the most important is self-stigma. ${ }^{22-25}$ Self-stigma is a continuing process during which the patient accepts negative evaluations of the social milieu, which are based on social preconceptions of being socially inferior because of the psychiatric disorder. ${ }^{26,27}$ Given existing social prejudices and stereotypes about psychiatric disorders, patients may feel incompetent, weak-willed, not strong enough to manage their lives, inferior, and burdening those around them, due to their psychological problems. ${ }^{28,29}$ Patients who agree with common prejudices about psychiatric patients do not believe in their own symptom improvement, feel depressed, and suffer from negative self-esteem. ${ }^{30-33}$ The worries of prejudices may influence the patient's efforts to find adequate treatment. ${ }^{34}$

Ociskova et $\mathrm{al}^{34}$ found that self-stigma was connected with the severity of depression, anxiety, and global evaluations of mental state at the beginning of treatment in individuals with NsD. Participants with a higher degree of self-stigma had lower progress during the therapy. Cinculova et $\mathrm{al}^{35}$ showed no correlation between self-stigma and age of disorder onset, age, or duration of the posthospitalization period. However, there were links between self-stigma and number of previous hospitalizations, severity of the disorder, discontinuation of medication, antidepressant dosage, and the number of psychiatrists visited by the patient. Additionally, self-stigma was negatively associated with adherence to treatment.

Also, Kamaradova et al, ${ }^{36}$ who inspected 332 patients with schizophrenia, bipolar disorders, depressive disorders, personality disorders, anxiety disorders, or substance-abuse disorders, found positive correlations between self-stigma, number of hospitalizations, severity of disease, lack of partnership, number of psychiatrists visited, doses of antidepressants, and nonadherence to treatment. A negative association was found with level of education.

Patients' efforts to avoid social stigma may lead to their denial of the fact that they are mentally ill, prioritizing 
somatic explanation of the causes of the problem, and trying to avoid or delay seeking appropriate psychiatric or psychological treatment. ${ }^{37-39}$ People with certain coping strategies, such as dissociation, are more inclined to feelings of guilt and shame. These individuals may be vulnerable to developing self-stigma as well, because the primary emotional source of self-stigma is the feeling of shame. ${ }^{40,41}$

Other critical psychological variables that have a substantial impact on QoL are coping strategies. ${ }^{22,42,43}$ Coping is a mental process of dealing with distress in different ways, with different approaches to the stressful situation, based on the individual's resources. As a component of individual tools, coping strategies represent efforts made by a patient to deal with distress. ${ }^{44}$ They should play an important role by connecting stressors and health consequences in both a physical and psychological way, and they could also relate to psychosocial factors, such as social support, subjective well-being, and resilience. ${ }^{45,46}$ Coping strategies can be divided according to different theories of stress management. In our study, we follow the parting of positive and negative management strategies in agreement with the authors of the Stress Coping Style Questionnaire (Strategie Zvládání Stresu [SVF] 78). Positive coping (Pos.Cop) and negative coping (Neg.Cop) mechanisms of patients reflected their maladaptive or adaptive problemsolving potential. ${ }^{47}$

Efficient and flexible strategies for managing stressful situations are reflected positively. Neg.Cop strategies include less flexible strategies in the context of the situation. ${ }^{48}$ Numerous maladaptive coping strategies are very effective in decreasing symptoms, at least in the short-term perspective. Unfortunately, after long-term use, they result in increased dysfunction and strengthening the disorder. ${ }^{49}$ Coping strategies may affect well-being, and are closely linked with QoL in many psychiatric disorders. ${ }^{50}$ The selection of coping strategies is influenced by the type of stressor, the individual's perception of the threat, available personal resources, and the strategies' effectiveness. ${ }^{51}$ Negative cognitive biases, negative self-esteem, and disturbed self-perception in coping with life may significantly weaken the possibility of using adaptive coping strategies. ${ }^{52}$

Models of NsD propose that inappropriate coping reactions to stressors play an essential part in their pathogenesis. Friedman et $\mathrm{al}^{53}$ pointed out that a positive approach to life may have a promising impact on QoL in connection with Pos. Cop (eg, more considerable effort to seek and accept support from social environment), but the question is whether patients who develop neurotic symptoms, such as anxiety in reaction to stress, can cope with stressful life events adaptively or not. Ociskova et $\mathrm{al}^{54}$ found that patients with an NsD who preferred maladaptive coping strategies profited from combined pharmacotherapy and psychotherapy considerably less than participants with more adaptive coping. Jacofsky et $\mathrm{al}^{49}$ described specific maladaptive coping strategies in different anxiety disorders that inhibited the individual's capability to disrupt paired associations between the situation and linked symptoms of anxiety, like sensitization, anxious avoidance, escape tendencies, negative reinforcement, and safety behavior.

\section{Aim and hypotheses}

This investigation was designed to measure self-stigma, QoL, and coping strategies of $\mathrm{NsD}$ outpatients as its first aim, followed by efforts to find out how self-stigma, QoL, and coping strategies are linked to one another, along with other clinical and demographic variables in outpatients with NsDs. Hypotheses were:

1. QoL will correlate negatively with self-stigma

2. QoL will correlate positively with Pos.Cop strategies, and negatively with Neg.Cop strategies

3. QoL will correlate negatively with the severity of the disorder

4. QoL will vary for different subgroups of $\mathrm{NsD}$

5. QoL will be significantly related to demographic factors, such as education, partnership, and employment

6. self-stigma will correlate positively with the severity of the disorder

7. self-stigma will correlate positively with Neg.Cop and negatively with Pos.Cop strategies

\section{Methods}

A total of 25 outpatient psychiatrists confirmed the diagnoses, including and excluding criteria, and administered questionnaires. The investigation was carried out between March 2014 and November 2015. Inclusion criteria were adult age, diagnosis of anxiety disorder, adjustment disorder, somatoform disorder, or OCD according to the ICD $10,{ }^{2}$ current mental state stabilized, and signing informal consent. Exclusion criteria were acute worsening of symptomatology, current hospitalization, current depressive disorder, mental retardation, substance-abuse disorder, and severe somatic illness. All patients attended the outpatient checkups and were treated with standard medication for anxiety and other NsDs according to the guidelines and principles of good clinical practice. 


\section{Assessment instruments}

The participants filled out several questionnaires during the routine psychiatric checkup.

\section{Quality of Life Satisfaction and Enjoyment Questionnaire}

The Quality of Life Satisfaction and Enjoyment Questionnaire (Q-LES-Q) comprises 93 articles divided into eight sections. ${ }^{55}$ Participants assess each section by picking out one number from a 5-point Likert scale according to agreement with the statement. Elaboration takes 20-30 minutes. Sections of the questionnaire are physical health, feelings, leisure, household, work, school/study, social relations, general, and a sum of QoL. The Czech version of the Q-LES-Q was validated by Müllerova. ${ }^{56}$ Analyses indicated high internal consistency (Cronbach's $\alpha=0.8-0.9$ ) and proved the stability of answers over time (test-retest) ${ }^{56}$ It is possible to get a total of 465 points from all domains together. The therapist guide contains average point values in individual domains, as well as the overall QoL of a healthy population for comparison with mentally ill patients. A healthy population in the therapist guide reached an average of 308 points, and $100 \%$ on total QoL corresponds to 465 points. ${ }^{56}$

\section{Internalized Stigma of Mental Illness scale}

The Internalized Stigma of Mental Illness (ISMI) scale has 29 items that assess five domains of self-stigma: alienation, perceived discrimination, stereotype endorsement, social withdrawal, and resistance to stigma. ${ }^{57,58}$ Internal consistency of the questionnaire is excellent. ${ }^{58}$ The Czech version $(\alpha=0.91)$ has excellent reliability (split-half method, Spearman-Brown coefficient 0.93) and test-retest consistency at 3 weeks after the initial assessment $(r=0.90, P<0.001){ }^{59}$ Therefore, when evaluating ISMI, total and partial scores are subtracted. Respondents can get a total of 116 points within the maximum number of self-stigmatizations.

\section{Clinical Global Impression}

The Clinical Global Impression (CGI) measure evaluates the global severity of psychopathology. ${ }^{60}$ The score is grounded on signs, behaviors, and functioning over the last 7 days, both observed and reported. It is assessed on a 7-point scale from 1 (normal) to 7 (most extremely ill). Assessment is accomplished by an experienced rater via the objective version (ObjCGI). Participants also evaluate themselves with the subjective version (SubjCGI). ${ }^{60}$ The scale has satisfactory internal consistency. ${ }^{61}$ Intraclass correlations lie in the range $0.88-0.92 .{ }^{61}$

\section{Stress Coping Style Questionnaire}

The Stress Coping Style Questionnaire ${ }^{47}$ (Strategie Zvládání Stresu [SVF] 78) contains 78 items distributed into 13 subscales, each representing a certain way of reacting to a stressful condition. The Czech version has a Cronbach's $\alpha$-value of $0.77-0.94 .^{62}$ Regular use of coping strategies is confirmed with $t=40-60::^{47} t>60$ indicates increased usage of coping strategies, and $t<40$ means decreased usage of suitable strategies. Neg.Cop strategies include escape tendency, perseveration, resignation, and self-accusation. Pos. Cop strategies include underestimation, guilt denial, diversion, compensatory satisfaction, situation control, reaction control, and positive self-instruction (Table 1). Only need for social support and active avoidance are not recognized in the total score. ${ }^{62}$

\section{Demographic questionnaire}

This form contained age, sex, marital status, education, employment status, type of pension, heredity, number of siblings, number of psychiatric hospitalizations, age at disorder onset, duration of attendance at outpatient treatment center, and current medication.

\section{Statistical evaluation and ethics}

GraphPad Prism 3 and SPSS 24.0 were used for statistical analysis. Demographic data and mean total scores were calculated using descriptive statistics. The distribution of data, means, medians, and SDs were defined. Means were compared using the unpaired $t$-test and the Mann-Whitney test. Relationships between specific sets were examined using correlation coefficients (Pearson or Spearman, according to data distribution) and linear regression. Connections between alternative variables were calculated using $\chi^{2}$ or Fisher's test (sex, marital status, partnership, education). Regression analysis was used to determine the significance of correlations of particular factors. For all statistical tests, a 5\% level of significance was established. To evaluate correlation coefficients, the Cohen model of correlation force developed for psychological testing (1988) was used, with a very weak correlation being $0-0.09$, weak correlation $0.09-0.29$, moderate relationship $0.30-0.49$, and strong relationship $0.50-1.00$. The study was approved by the local ethics committee of the University Hospital Olomouc. All participants provided signed informed consent. The study was performed in accordance with the Declaration of Helsinki and guidelines for good clinical practice. ${ }^{63}$ 
Table I Coping strategies according to the SVF78

\begin{tabular}{|c|c|}
\hline & Specification \\
\hline Underestimation (Pos.Cop) & $\begin{array}{l}\text { Tendency to attribute lower levels of stress to oneself compared to others; leads to underestimation of the } \\
\text { reaction or favorable evaluation of it }\end{array}$ \\
\hline Guilt denial (Pos.Cop) & Not taking responsibility for one's burdens; emphasizes a defensive approach \\
\hline Diversion (Pos.Cop) & $\begin{array}{l}\text { Diversion from stressful situations and activities or diversion to situations unrelated to the stressful situation; } \\
\text { tendency to avert load or induce states of relieving stress, eg, using humor }\end{array}$ \\
\hline $\begin{array}{l}\text { Compensatory satisfaction } \\
\text { (Pos.Cop) }\end{array}$ & $\begin{array}{l}\text { Tendency to draw attention to positive activities; discussions focused on positive emotions not related to } \\
\text { stress, leading to self-empowerment by external rewards (eg, food, shopping) }\end{array}$ \\
\hline Situation control (Pos.Cop) & $\begin{array}{l}\text { Tendency to gain control of the situation, situation analysis, planning, action, and active intervention in the } \\
\text { situation; this form of stress management is considered constructive }\end{array}$ \\
\hline Reaction control (Pos.Cop) & Effort to control one's own reactions, not allowing them to overwhelm the situation \\
\hline $\begin{array}{l}\text { Positive self-instruction } \\
\text { (Pos.Cop) }\end{array}$ & $\begin{array}{l}\text { Tendency to embrace responsibility for oneself, effort to gain and maintain a positive attitude, perseverance, } \\
\text { and ability to sustain }\end{array}$ \\
\hline $\begin{array}{l}\text { Need for social support } \\
\text { (neutral) }\end{array}$ & $\begin{array}{l}\text { Tendency to seek support and assistance in the social arena, either in the form of passive-resignation attitudes } \\
\text { or actively seeking concrete support in solving the problem }\end{array}$ \\
\hline Active avoidance (neutral) & Tendency to avoid the actual problem and attempts to avoid other similar situations \\
\hline Escape tendency (Neg.Cop) & $\begin{array}{l}\text { Tendency to escape from maximum-stress situations, which leads to decreased ability to face and solve them; } \\
\text { this strategy is a form of resignation }\end{array}$ \\
\hline Perseveration (Neg.Cop) & $\begin{array}{l}\text { Increased and prolonged reflection on the situation and overthinking the stress; negative thoughts on the stress } \\
\text { situation are intrusive and occupy the capacity of thought processes }\end{array}$ \\
\hline Resignation (Neg.Cop) & $\begin{array}{l}\text { Subjective feelings of lack of ability to handle stressful situations; resignation is accompanied by feelings of } \\
\text { helplessness and hopelessness, and the individual surrenders without even an attempt to overcome the } \\
\text { problem, believing that the situation is unsolvable, or that they cannot cope with it }\end{array}$ \\
\hline Self-blame (Neg.Cop) & Tendency to depressive mood and to attribute errors related to stressful situations to oneself \\
\hline
\end{tabular}

Abbreviations: SVF, Strategie Zvládání Stresu (Stress Coping Style Questionnaire); Pos.Cop, positive coping; Neg.Cop, negative coping.

\section{Results}

\section{Sample characteristics}

A total of 153 outpatients with NsD diagnosed according to ICD10 criteria completed the scales $(73.9 \%$ women $)$ and questionnaires (150 SVF78, 152 ISMI, 153 Q-LES-Q). The mean age was $48.72 \pm 13.41$ years. The majority of the patients were married (49.0\%). Almost half the patients had secondary education (47.1\%) and around a fifth university level (18.3\%, Table 2). Family psychiatric history was recognized in 29 participants (34.3\%), and $57.5 \%$ of patients were employed. In total, $22.8 \%$ of participants received disability benefits (full disability benefits [7.8\%] and partial disability benefits [14.4\%]), 19.6\% were retired, and $19.0 \%$ of the patients had been hospitalized (Table 2).

Patients with NsD were assessed by their psychiatrist as mildly mentally ill (2.67 \pm 1.19$)$ [according ObjCGI]. Similarly, the severity of mental illness was also evaluated by patients (2.31 \pm 1.17 , Table 2) [according SubjCGI]. Of the total 465 points of the QoL, patient scores averaged 287.9 55.4 (62\%, Table 2). ISMI total score averaged $53.49 \pm 11.84(t=43)$, with lower scores on ISMI - perceived discrimination $(7.76 \pm 2.58$,
Table 2 Demographic and clinical characteristics of the patients

\begin{tabular}{|l|l|}
\hline & Patients (n= I 53) \\
\hline Age, years & $48.72 \pm|3.4|$ \\
\hline Sex (M:F) & $39: 114$ \\
\hline Age at disease onset, years & $38.60 \pm 16.02$ \\
\hline Lifetime duration of treatment, years & $9.27 \pm 10.12$ \\
\hline Number of hospitalizations & $0.32 \pm 0.82$ \\
\hline Education & \\
Elementary & 10 \\
Vocational training & 40 \\
Secondary school & 72 \\
University & 28 \\
Not completed & 3 \\
\hline Marital status & \\
Single & 32 \\
Married & 75 \\
Divorced & 39 \\
Widowed & 9 \\
Not completed & 7 \\
\hline Partner & \\
No & 70 \\
Yes & 35 \\
Not completed & 48 \\
\hline
\end{tabular}

(Continued) 
Table 2 (Continued)

\begin{tabular}{|l|l|}
\hline & Patients (n=I53) \\
\hline Employment & \\
No & 64 \\
Yes & 88 \\
Not completed & 1 \\
\hline ObjCGI severity & $2.67 \pm 1.19$ \\
\hline SubjCGI severity & $2.31 \pm 1.17$ \\
\hline Physical health (max 65p) & $40.68 \pm 8.74$ \\
Feelings (max 70p) & $49.54 \pm 9.86$ \\
Work (max 65p) & $35.21 \pm 19.27$ \\
Household (max 50p) & $39.53 \pm 9.37$ \\
School/study (max 50p) & $11.75 \pm 6.47$ \\
Leisure (max 30p) & $20.95 \pm 5.26$ \\
Social activities (max 55p) & $38.86 \pm 7.78$ \\
General (max 80p) & $53.78 \pm 10.54$ \\
Sum Q-LES-Q (max 465p) & $287.9 \pm 55.4$ \\
Sum Q-LES-Q & $61.91 \% \pm 11.91 \%$ \\
\hline Alienation & $10.93 \pm 3.85$ \\
Stereotype endorsement & $11.52 \pm 2.98$ \\
Perceived discrimination & $7.76 \pm 2.58$ \\
Social withdrawal & $10.49 \pm 3.33$ \\
Stigma resistance & $12.53 \pm 2.41$ \\
ISMl overall score & $53.49 \pm 11.84$ \\
\hline Positive coping & $12.86 \pm 3.19$ \\
Negative coping & $13.11 \pm 3.88$ \\
Underestimation & $8.83 \pm 4.35$ \\
Guilt denial & $11.99 \pm 4.09$ \\
Diversion & $13.09 \pm 4.62$ \\
Compensatory satisfaction & $11.98 \pm 4.69$ \\
Situation control & $14.73 \pm 4.05$ \\
Reaction control & $15.47 \pm 3.99$ \\
Positive self-instruction & $14.56 \pm 4.62$ \\
Need for social support & $13.31 \pm 5.10$ \\
Active avoidance & $15.46 \pm 3.95$ \\
Escape tendency & $12.67 \pm 4.37$ \\
Rumination/perseveration & $16.22 \pm 5.38$ \\
Resignation & $11.24 \pm 4.68$ \\
Self-accusation & $12.14 \pm 5.19$ \\
\hline Abrevtionsi ObjCGl, & \\
\hline
\end{tabular}

Abbreviations: ObjCGI, objective Clinical Global Impression; SubjCGI, subjective CGI; Q-LES-Q, Quality of Life Enjoyment and Satisfaction Questionnaire; ISMI, Internalized Stigma of Mental Illness; p, points.

Table 2). Use of Pos.Cop strategies showed $t=50(12.86 \pm 3.19)$, and Neg.Cop strategies $t=60(13.11 \pm 3.88$, Table 2$)$.

\section{Diagnostic subgroups}

The sample was diagnostically heterogeneous in this category of mental illness, comprising six subgroups of NsD diagnoses: panic disorder and/or agoraphobia, social phobia, GAD and mixed anxiety-depression disorder, adjustment disorder, somatoform disorders, and OCD. The diagnostic subgroups differed significantly only in age and use of Neg. Cop strategies, but not in other measured clinical and psychological variables (Table 3). Considering Neg.Cop, in which the disease groups differed significantly, patients with social phobia used the highest use of Neg.Cop strategies from all NsD groups. Patients with panic disorder and agoraphobia had the lowest average use of Neg.Cop strategies (Table 3).

\section{Treatment}

A total of 147 patients used psychopharmacological drugs (96.1\%), only six did not use any psychiatric medication (3.9\%), while 139 (90.9\%) reported using prescribed antidepressant medication. Most patients took the medication in prescribed dosages regularly $(90.2 \%)$, and nine $(6.0 \%)$ irregularly (occasionally forgetting to take it). Individual doses were not checked in this investigation.

\section{Demographic factors, quality of life, self- stigma and coping strategies}

There were no differences between the sexes in disorder severity (measured by ObjCGI or SubjCGI), Q-LES-Q total, ISMI total, or Pos.Cop and Neg.Cop strategies (Table 4).

No statistically significant differences were found among subgroups defined by education in connection with disorder severity evaluated by ObjCGI, SubjCGI, self-stigma measured by ISMI total, Pos.Cop, or Neg.Cop. Nevertheless, there was significant difference among educational subgroups in Q-LES-Q (one-way ANOVA, $F_{148}=3.11 ; P<0.05$ ), more concretely among elementary and university education (Table 4). Partnership and employment have no significant influence on subjectively or objectively evaluated severity of the disorder assessed by SubjCGI and ObjCGI, self-stigma assessed by ISMI total, Pos.Cop, or Neg.Cop. QoL represented by Q-LES-Q total showed a statistically significant difference between unemployed and employed participants, while partnership had no significant effect on QoL (Table 4).

\section{Quality of life}

Subgroups of participants classified by diagnosis did not differ significantly in relation to the severity of the disorder (Kruskal-Wallis test), ISMI (one-way ANOVA), or Pos.Cop variables.

Relationships between QOL and demographic and clinical variables

Many correlations were found between domains of QoL and clinical and demographic factors (Table 5). Physical health and feelings correlated negatively with ObjCGI and SubjCGI. The work domain correlated negatively with age, onset of disorder, and ObjCGI. All domains of the Q-LES-Q correlated negatively with ObjCGI and SubjCGI, except for 
Table 3 Differences in assessment scales according to diagnoses

\begin{tabular}{|c|c|c|c|c|c|c|}
\hline & ObjCGI & SubjCGI & ISMI total & Pos.Cop & Neg.Cop & Q-LES-Q total \\
\hline $\begin{array}{l}\text { Panic disorder with or without } \\
\text { agoraphobia }(n=2 I)\end{array}$ & $2.52 \pm 0.98$ & $1.95 \pm 0.67$ & $50.4 \pm 7.9$ & $13.3 \pm 2.3$ & $10.9 \pm 3.7$ & $310.6 \pm 37.3$ \\
\hline Social phobia $(n=8)$ & $3.38 \pm 0.74$ & $2.63 \pm 1.41$ & $54.1 \pm 10.9$ & $10.7 \pm 2.4$ & $15.7 \pm 3.4$ & $272.4 \pm 50.2$ \\
\hline $\begin{array}{l}\text { Generalized anxiety disorder and mixed } \\
\text { anxiety-depressive disorder }(n=65)\end{array}$ & $2.77 \pm 1.24$ & $2.23 \pm 1.22$ & $52.9 \pm 12.4$ & $13.2 \pm 3.7$ & $13.1 \pm 3.5$ & $293.3 \pm 53.4$ \\
\hline Adjustment disorders $(n=33)$ & $2.39 \pm 1.30$ & $2.67 \pm 1.32$ & $56.8 \pm 14.0$ & $13.0 \pm 3.1$ & $13.7 \pm 4.5$ & $279.5 \pm 46.2$ \\
\hline Somatoform disorders $(n=20)$ & $2.56 \pm 1.28$ & $2.10 \pm 1.07$ & $52.4 \pm 10.2$ & $12.3 \pm 2.6$ & $13.9 \pm 3.6$ & $278.3 \pm 57.0$ \\
\hline Obsessive-compulsive disorder $(n=6)$ & $3.17 \pm 0.75$ & $2.67 \pm 0.52$ & $55.7 \pm 10.8$ & $11.9 \pm 2.3$ & $12.0 \pm 3.3$ & $298.2 \pm 41.9$ \\
\hline Statistics & NS* & NS* & $F_{151}=0.896, \mathrm{NS}^{\#}$ & $F_{149}=1.19, \mathrm{NS}^{\#}$ & $\begin{array}{l}F_{149}=2.56, P<0.05 \\
\text { (panic vs social } \\
\text { phobia, } P<0.05)^{\#}\end{array}$ & $F_{152}=1.16, \mathrm{NS}^{\#}$ \\
\hline
\end{tabular}

Notes: *Kruskal-Wallis test; *one-way ANOVA.

Abbreviations: ObjCGI, objective Clinical Global Impression; SubjCGI, subjective CGI; ISMI, Internalized Stigma of Mental Illness; Pos.Cop, positive coping; Neg.Cop, negative coping; Q-LES-Q, Quality of Life Enjoyment and Satisfaction Questionnaire; NS, not significant.

Table 4 Differences according to demographic factors in assessment scales

\begin{tabular}{|c|c|c|c|c|c|c|}
\hline & ObjCGI & SubjCGI & ISMI total & Pos.Cop & Neg.Cop & Q-LES-Q total \\
\hline $\begin{array}{l}\text { Sex } \\
\text { Male }(n=39) \\
\text { Female }(n=1 \mid 4)\end{array}$ & $\begin{array}{l}2.72 \pm 1.12 \\
2.66 \pm 1.23\end{array}$ & $\begin{array}{l}2.26 \pm 1.02 \\
2.33 \pm 1.22\end{array}$ & $\begin{array}{l}52.8 \pm 10.1 \\
53.7 \pm 12.4\end{array}$ & $\begin{array}{l}12.7 \pm 3.4 \\
12.9 \pm 3.1\end{array}$ & $\begin{array}{l}12.3 \pm 3.7 \\
13.4 \pm 3.9\end{array}$ & $\begin{array}{l}289.2 \pm 39.4 \\
287.5 \pm 50.0\end{array}$ \\
\hline Statistics & NS* & NS* & $t_{150}=0.44, \# \mathrm{NS}$ & $t_{148}=0.27$, $\mathrm{NS}$ & $t_{148}=1.5, \# \mathrm{NS}$ & $t_{151}=0.17, \# \mathrm{NS}$ \\
\hline $\begin{array}{l}\text { Education } \\
\text { Elementary }(n=10) \\
\text { Vocational training }(n=40) \\
\text { Secondary school }(n=72) \\
\text { University }(n=28)\end{array}$ & $\begin{array}{l}2.30 \pm 1.25 \\
2.85 \pm 1.08 \\
2.68 \pm 1.27 \\
2.46 \pm 1.04\end{array}$ & $\begin{array}{l}2.20 \pm 1.32 \\
2.33 \pm 1.14 \\
2.29 \pm 1.14 \\
2.21 \pm 0.92\end{array}$ & $\begin{array}{l}53.3 \pm 16.6 \\
53.8 \pm 11.5 \\
53.5 \pm 11.8 \\
50.9 \pm 8.5\end{array}$ & $\begin{array}{l}13.7 \pm 4.0 \\
12.8 \pm 3.3 \\
12.8 \pm 3.1 \\
12.8 \pm 3.3\end{array}$ & $\begin{array}{l}13.0 \pm 4.5 \\
13.1+3.7 \\
13.1 \pm 3.8 \\
12.8 \pm 4.1\end{array}$ & $\begin{array}{l}261.9 \pm 54.9 \\
283.9 \pm 46.8 \\
290.7 \pm 49.7 \\
312.1 \pm 49.1\end{array}$ \\
\hline Statistics & NS** & NS** & $F_{148}=0.42, \ldots N S$ & $F_{148}=0.26,{ }^{\#} \mathrm{NS}$ & $F_{148}=0.05, \ldots N S$ & $\begin{array}{l}F_{148}=3.1 \mathrm{I},{ }^{\#} P<0.05 \text { (elementary } \\
\text { vs university, } P<0.05 \text { ) }\end{array}$ \\
\hline $\begin{array}{l}\text { Partner } \\
\text { No }(n=49) \\
\text { Yes }(n=98)\end{array}$ & $\begin{array}{l}2.78 \pm 1.34 \\
2.62 \pm 1.11\end{array}$ & $\begin{array}{l}2.41 \pm 1.46 \\
2.26 \pm 1.03\end{array}$ & $\begin{array}{l}52.3 \pm 14.2 \\
54.0 \pm 10.7\end{array}$ & $\begin{array}{l}13.1 \pm 3.3 \\
12.9 \pm 3.1\end{array}$ & $\begin{array}{l}12.8 \pm 4.1 \\
13.1 \pm 3.8\end{array}$ & $\begin{array}{l}298.0 \pm 50.9 \\
287.0 \pm 50.8\end{array}$ \\
\hline Statistics & NS* & NS* & $t_{144}=0.79, \# \mathrm{NS}$ & $t_{142}=0.33$, \# NS & $t_{142}=0.34, \# \mathrm{NS}$ & $t_{144}=1.24, \# \mathrm{NS}$ \\
\hline $\begin{array}{l}\text { Employment } \\
\text { No }(n=64) \\
\text { Yes }(n=88)\end{array}$ & $\begin{array}{l}2.88 \pm 1.18 \\
2.52 \pm 1.20\end{array}$ & $\begin{array}{l}2.47 \pm 1.38 \\
2.19 \pm 0.98\end{array}$ & $\begin{array}{l}55.7 \pm 13.2 \\
52.1 \pm 10.5\end{array}$ & $\begin{array}{l}12.7 \pm 3.6 \\
12.9 \pm 2.9\end{array}$ & $\begin{array}{l}13.2 \pm 4.3 \\
13.1 \pm 3.5\end{array}$ & $\begin{array}{l}267.9 \pm 43.4 \\
304.4 \pm 48.2\end{array}$ \\
\hline Statistics & NS* & NS* & $t_{149}=1.8$, N NS & $t_{147}=0.34, \# \mathrm{NS}$ & $t_{147}=0.22, \# \mathrm{NS}$ & $t_{149}=4.797, \# P<0.0001$ \\
\hline
\end{tabular}

Notes: *Mann-Whitney test; "unpaired $t$-test; **Kruskal-Wallis test; "\#one-way ANOVA.

Abbreviations: ObjCGI, objective Clinical Global Impression; SubjCGI, subjective CGI; ISMI, Internalized Stigma of Mental Illness; Pos.Cop, positive coping; Neg.Cop, negative coping; Q-LES-Q, Quality of Life Enjoyment and Satisfaction Questionnaire; NS, not significant.

the domain school/study. The sum of Q-LES-Q correlated negatively with SubjCGI and ObjCGI, but not other demographic factors (Table 5).

\section{Relationships between self-stigma and quality of life}

All Q-LES-Q domains significantly negatively correlated with all ISMI domains, except school/study. Strong correlations were mainly between social withdrawal and the Q-LESQ total score. The domain of feelings correlated strongly with alienation and stereotype endorsement. (Table 6).

\section{Relationships between coping strategies and quality} of life

The sum of Pos.Cop significantly positively correlated with all Q-LES-Q domains except school/study. Q-LES-Q domains correlated negatively with Neg.Cop, except for the domains work and school/study (Table 7). Q-LES-Q total has either statistically significant positive/negative correlations with all domains, except for the strategy called guilt denial (not taking responsibility for burdens, emphasizes defensive approach). 
Table 5 Correlations between QoL and demographic or clinical variables

\begin{tabular}{|c|c|c|c|c|c|c|}
\hline & Age & Disorder onset & Disorder duration & ObjCGI & SubjCGI & Hospitalizations \\
\hline Physical health & $-0.06^{P}$ & $0.01^{P}$ & $-0.19^{5}$ & $-0.21^{\mathrm{S} * *}$ & $-0.30^{\text {S*** }}$ & $-0.19^{p *}$ \\
\hline Feelings & $0.04^{P}$ & $0.09^{p}$ & $-0.11^{s}$ & $-0.18^{5 *}$ & $-0.5 I^{\text {S*** }}$ & $-0.12^{P}$ \\
\hline Work & $-0.35^{5 * * *}$ & $-0.22^{5 * * *}$ & $-0.09^{\mathrm{s}}$ & $-0.17^{5 *}$ & $-0.15^{s}$ & $-0.01^{\mathrm{s}}$ \\
\hline Household & $0.19^{5 *}$ & $0.24^{5 * *}$ & $-0.0 \mathrm{I}^{\mathrm{s}}$ & $-0.21^{\mathrm{s} * *}$ & $-0.32^{\text {s*** }}$ & $-0.12^{\mathrm{s}}$ \\
\hline School/study & $-0.22^{5 * *}$ & $-0.19^{5 *}$ & $-0.0 \mathrm{I}^{\mathrm{s}}$ & $0.02^{\mathrm{s}}$ & $0.14^{\mathrm{s}}$ & $-0.04^{\mathrm{s}}$ \\
\hline Leisure time & $-0.17^{5 *}$ & $0.15^{\mathrm{s}}$ & $-0.07^{\mathrm{s}}$ & $-0.36^{\text {s** }}$ & $-0.4 I^{S * * *}$ & $-0.15^{\mathrm{s}}$ \\
\hline Social activities & $0.0 \mathrm{I}^{\mathrm{P}}$ & $0.1 I^{P}$ & $0.05^{\mathrm{s}}$ & $-0.27^{\text {S*** }}$ & $-0.36^{\text {s*** }}$ & $-0.02^{P}$ \\
\hline General & $-0.03^{P}$ & $0.07^{P}$ & $-0.10^{s}$ & $-0.24^{\mathrm{S**}}$ & $-0.32^{\text {S*** }}$ & $-0.04^{P}$ \\
\hline Sum Q-LES-Q & $-0.14^{\mathrm{s}}$ & $-0.02^{P}$ & $-0.14^{\mathrm{s}}$ & $-0.28^{5 * * *}$ & $-0.47^{5 * * *}$ & $-0.14^{\mathrm{P}}$ \\
\hline
\end{tabular}

Notes: $* P<0.05 ; * * P<0.01 ; * * * P<0.001$.

Abbreviations: ObjCGI, objective Clinical Global Impression; SubjCGI, subjective CGI; Q-LES-Q, Quality of Life Enjoyment and Satisfaction Questionnaire; P, Pearson's $r$; S, Spearman's $r$.

Table 6 Relationships between Q-LES-Q domains and self-stigma

\begin{tabular}{|c|c|c|c|c|c|c|}
\hline & $\begin{array}{l}\text { Overall ISMI } \\
\text { score }\end{array}$ & Alienation & $\begin{array}{l}\text { Stereotype } \\
\text { endorsement }\end{array}$ & $\begin{array}{l}\text { Perceived } \\
\text { discrimination }\end{array}$ & $\begin{array}{l}\text { Social } \\
\text { withdrawal }\end{array}$ & \begin{tabular}{|l} 
Stigma \\
resistance
\end{tabular} \\
\hline Physical health & $-0.50^{\text {P*** }}$ & $-0.44^{\text {s*** }}$ & $-0.45^{\mathrm{P} * * *}$ & $-0.46^{\text {S*** }}$ & $-0.46^{\text {S*** }}$ & $-0.25^{5 * * *}$ \\
\hline Feelings & $-0.65^{P * * *}$ & $-0.57^{5 * * *}$ & $-0.57^{\mathrm{P} * * *}$ & $-0.52^{\text {s*** }}$ & $-0.59^{5 * * *}$ & $-0.36^{\text {s*** }}$ \\
\hline Work & $-0.26^{5 * * *}$ & $-0.17^{5 *}$ & $-0.18^{5 *}$ & $-0.21^{\mathrm{s} * *}$ & -0.29 s*** & $-0.23^{\text {s** }}$ \\
\hline Household & $-0.50^{\text {S*** }}$ & $-0.46^{5 * * * *}$ & $-0.46^{\text {5*** }}$ & $-0.46^{\text {5*** }}$ & $-0.49^{\text {s*** }}$ & $-0.14^{5 *}$ \\
\hline School/study & $-0.00^{\mathrm{s}}$ & $-0.20^{\mathrm{s}}$ & $-0.09^{\mathrm{s}}$ & $-0.07^{s}$ & 0.04 & $-0.03^{\mathrm{s}}$ \\
\hline Leisure & $-0.45^{5 * * *}$ & $-0.44^{\text {s*** }}$ & $-0.41^{\mathrm{S} * * *}$ & $-0.34^{\text {S*** }}$ & $-0.39^{5 * * *}$ & $-0.32^{\text {s*** }}$ \\
\hline Social activities & $-0.57^{\text {P**** }}$ & $-0.48^{\text {S*** }}$ & $-0.42^{\text {P*** }}$ & $-0.43^{\text {S**** }}$ & $-0.50^{5 * * *}$ & $-0.34^{\text {S*** }}$ \\
\hline General & $-0.55^{\text {P*** }}$ & $-0.55^{5 * * *}$ & $-0.44^{\mathrm{P} * * *}$ & $-0.49^{\text {S*** }}$ & $-0.54^{\text {s*** }}$ & $-0.32^{\text {S*** }}$ \\
\hline Sum Q-LES-Q & $0.59^{\mathrm{P} * * *}$ & $-0.55^{\text {5*** }}$ & $-0.50^{\mathrm{P} * * *}$ & $-0.5 I^{\text {S*** }}$ & $-0.61^{\text {I*** }}$ & $-0.37^{\text {S*** }}$ \\
\hline
\end{tabular}

Notes: $* P<0.05 ; * * P<0.01 ; * * * P<0.001$.

Abbreviations: Q-LES-Q, Quality of Life Enjoyment and Satisfaction Questionnaire; ISMI, Internalized Stigma of Mental IIIness; P, Pearson's r; S, Spearman's $r$.

Table 7 Relationships among Q-LES-Q domains and coping strategies

\begin{tabular}{|c|c|c|c|c|c|c|c|c|c|}
\hline & $\begin{array}{l}\text { Physical } \\
\text { health }\end{array}$ & Feelings & Work & Household & $\begin{array}{l}\text { School/ } \\
\text { study }\end{array}$ & Leisure & $\begin{array}{l}\text { Social } \\
\text { activities }\end{array}$ & General & $\begin{array}{l}\text { Sum } \\
\text { Q-LES-Q }\end{array}$ \\
\hline Pos.Cop & $0.28^{\mathrm{P} * * *}$ & $0.33^{\mathrm{P} * * *}$ & $0.19^{5 *}$ & $0.35^{5 * * *}$ & 0.06 & $0.27^{5 * * *}$ & $0.3 I^{\mathrm{P} * * * *}$ & $0.3 I^{\mathrm{P} * * *}$ & $0.40^{\mathrm{P} * * *}$ \\
\hline Neg.Cop & $-0.38^{\mathrm{P} * * *}$ & $-0.47^{\text {P**** }}$ & -0.15 & $-0.34^{5 * * *}$ & -0.09 & $-0.33^{\text {s*** }}$ & $-0.33^{\mathrm{P} * * *}$ & $-0.38^{\mathrm{P} * * *}$ & $-0.38^{\mathrm{P} * * *}$ \\
\hline Underestimation & 0.04 & 0.16 & 0.06 & $0.26^{5 * *}$ & 0.04 & 0.16 & 0.11 & $0.16^{\mathrm{P} *}$ & $0.19^{P *}$ \\
\hline Guilt denial & $0.18^{\mathrm{P} *}$ & 0.10 & 0.02 & $0.18^{5 *}$ & -0.09 & $0.19^{5 *}$ & 0.03 & $0.19^{P *}$ & 0.13 \\
\hline Diversion & $0.25^{\mathrm{P} * *}$ & $0.3 \mathrm{I}^{\mathrm{P} * * *}$ & 0.05 & $0.37^{5 * * * *}$ & 0.06 & $0.29^{5 * * *}$ & $0.36^{\mathrm{P} * * *}$ & $0.25^{\mathrm{p} * *}$ & $0.36^{\mathrm{P} * * *}$ \\
\hline Compensatory satisfaction & $0.19^{\mathrm{P} *}$ & 0.15 & 0.08 & $0.18^{5 *}$ & 0.07 & 0.12 & $0.23^{\mathrm{P} * *}$ & 0.12 & $0.24^{\mathrm{P} * *}$ \\
\hline Situation control & 0.13 & $0.17^{\mathrm{P} *}$ & $0.24^{5 * *}$ & $0.21^{\mathrm{s} * *}$ & -0.01 & $0.21^{5 *}$ & $0.20^{\mathrm{P} *}$ & $0.20^{\mathrm{p} * *}$ & $0.23^{\mathrm{P} * *}$ \\
\hline Reaction control & $0.29^{\mathrm{P} * * *}$ & $0.34^{\mathrm{P} * * *}$ & $0.19^{5 *}$ & $0.27^{5 * * *}$ & 0.15 & $0.23^{\text {s** }}$ & $0.28^{\mathrm{P} * * * *}$ & $0.28^{\mathrm{P} * * *}$ & $0.36^{\mathrm{P} * * *}$ \\
\hline Positive self-instruction & $0.3 I^{\mathrm{P} * * * *}$ & $0.42^{\text {P*** }}$ & $0.21^{\mathrm{s} * *}$ & $0.34^{5 * * * *}$ & 0.11 & $0.27^{5 * * *}$ & $0.33^{\mathrm{P} * * *}$ & $0.32^{\mathrm{P} * * *}$ & $0.39^{\mathrm{P} * * *}$ \\
\hline Need for social support & 0.10 & 0.03 & 0.06 & $0.195 *$ & 0.01 & 0.03 & $0.30^{\mathrm{P} * * *}$ & $0.17^{\mathrm{P} *}$ & $0.16^{\mathrm{P} *}$ \\
\hline Active avoidance & 0.12 & 0.04 & 0.09 & 0.14 & 0.10 & 0.04 & 0.04 & 0.08 & $0.20^{P *}$ \\
\hline Escape tendency & $-0.33^{\mathrm{P} * * *}$ & $-0.38^{\mathrm{P} * * *}$ & -0.10 & $-0.29^{5 * * *}$ & -0.09 & $-0.19^{\text {S*** }}$ & $-0.20^{\mathrm{P} *}$ & $-0.3 \mathrm{I}^{\mathrm{P} * * *}$ & $-0.20^{\mathrm{P} *}$ \\
\hline Rumination/perseveration & $-0.25^{\mathrm{P} * *}$ & $-0.33^{\mathrm{P} * * *}$ & -0.09 & $-0.25^{5 * *}$ & -0.13 & $-0.21^{5 *}$ & $-0.27^{\mathrm{P} * * *}$ & $-0.27^{\mathrm{P} * * *}$ & $-0.30^{\text {P**** }}$ \\
\hline Resignation & $-0.37^{\text {P*** }}$ & $-0.49^{\text {P*** }}$ & $-0.25^{5 * *}$ & $-0.36^{\text {5*** }}$ & -0.13 & $-0.34^{\text {S*** }}$ & $-0.39^{\mathrm{P} * * *}$ & $-0.39^{\text {P*** }}$ & $-0.42^{\mathrm{P} * * *}$ \\
\hline Self-accusation & $-0.28^{\mathrm{P} * * *}$ & $-0.35^{\mathrm{P} * * *}$ & -0.07 & $-0.25^{5 * *}$ & 0 & $-0.29^{5 * * *}$ & $-0.26^{\mathrm{P} * * *}$ & $-0.32^{\text {P*** }}$ & $-0.33^{\mathrm{P} * * *}$ \\
\hline
\end{tabular}

Notes: $* P<0.05 ; * * P<0.01 ; * * * P<0.001$.

Abbreviations: Q-LES-Q, Quality of Life Enjoyment and Satisfaction Questionnaire; Pos.Cop, positive coping (SVF78); Neg.Cop, negative coping (SVF78); P, Pearson's r; S, Spearman's r; SVF, Strategie Zvládání Stresu (Stress Coping Style Questionnaire). 


\section{Self-stigma}

Relationships between demographic or clinical variables and self-stigma

Self-stigma measured by an overall score of ISMI significantly correlated positively with SubjCGI - severity, ObjCGI - severity, and number of hospitalizations. The onset of the disorder correlated only with alienation, duration of disease did not correlate with any ISMI domains, and number of hospitalizations correlated positively with alienation, stereotype endorsement, perceived discrimination, and social withdrawal, but did not correlate with stigma resistance (Table 8). SubjCGI and ObjCGI correlated significantly positively with all domains of ISMI except stigma resistance.

\section{Relationships between coping strategies and self-stigma}

The sum of Pos.Cop strategies correlated negatively with all domains of self-stigma, just as Neg.Cop strategies correlated positively with all domains of self-stigma measured by ISMI (Table 8 ).

\section{Regression analyses}

Because several factors were related to the sum of the Q-LES$\mathrm{Q}$, multiple stepwise-regression analyses were performed to detect the major critical factors linked to QoL in outpatients with NsD. The dependent variable was Q-LES-Q total. The independent variables were partnership, diagnostic subgroup, number of hospitalizations, ObjCGI, SubjCGI, Pos. Cop, Neg.Cop, employment, and ISMI total. Six steps of backward-regression analysis identified the most significant factors connected with Q-LES-Q total. Those were Pos.Cop, employment, and overall ISMI rating, which explained 32.9\% of Q-LES-Q total scores (Table 9).

According to the hypothesis, self-stigma was significantly related to many factors, so we performed the multiple-regression analyses for this as well. Independent factors included age, sex, partnership, education, employment, retirement, onset of disorder, duration of disorder, number of hospitalizations, Q-LES-Q total, Pos.Cop, and Neg.Cop. The most significant factors connected to self-stigma were SubjCGI, Pos.Cop, and Q-LES-Q total, which explained $44.5 \%$ of ISMI total $\left(F_{88}=18.673, P<0.001\right.$; Table 9).

Table 8 Relationships between self-stigma and demographic, clinical variables, and coping strategies

\begin{tabular}{|c|c|c|c|c|c|c|}
\hline & ISMI total & Alienation & $\begin{array}{l}\text { Stereotype } \\
\text { endorsement }\end{array}$ & $\begin{array}{l}\text { Perceived } \\
\text { discrimination }\end{array}$ & $\begin{array}{l}\text { Social } \\
\text { withdrawal }\end{array}$ & $\begin{array}{l}\text { Stigma } \\
\text { resistance }\end{array}$ \\
\hline Age & -0.03 & -0.05 & 0.05 & -0.06 & 0.06 & -0.05 \\
\hline Onset of disorder & -0.06 & $-0.19^{5 * *}$ & -0.10 & -0.08 & 0 & 0.05 \\
\hline Duration of disorder & 0.06 & -0.10 & 0.08 & 0.02 & 0.06 & 0 \\
\hline Number of hospitalizations & $0.23^{5 * *}$ & $0.22^{5 * *}$ & $0.19^{5 *}$ & $0.18^{5 *}$ & $0.22^{5 * *}$ & -0.02 \\
\hline ObjCGI - severity & $0.24^{\text {S** }}$ & $0.27^{5 * * *}$ & $0.16^{5 *}$ & $0.19^{5 *}$ & $0.21^{5 *}$ & 0.13 \\
\hline SubjCGI - severity & $0.43^{\text {s*** }}$ & $0.50^{5 * * *}$ & $0.37^{5 * * *}$ & $0.36^{5 * * *}$ & $0.43^{\text {S*** }}$ & 0.12 \\
\hline Pos.Cop & $-0.5 I^{P * * *}$ & $-0.52^{5 * * *}$ & $-0.40^{5 * * *}$ & $-0.40^{5 * * *}$ & $-0.45^{5 * * *}$ & $-0.34^{5 * * *}$ \\
\hline Neg.Cop & $0.48^{\mathrm{P} * * *}$ & $0.53^{5 * * *}$ & $0.45^{5 * * *}$ & $0.36^{5 * * *}$ & $0.48^{\mathrm{S} * * *}$ & $0.30^{5 * * *}$ \\
\hline Underestimation (Pos.Cop) & $-0.30^{\mathrm{P} * * *}$ & $-0.42^{\text {S**** }}$ & $-0.29^{5 * * *}$ & $-0.23^{5 * *}$ & $-0.29^{5 * * *}$ & $-0.19^{5 *}$ \\
\hline Guilt denial (Pos.Cop) & $-0.27^{\text {P*** }}$ & $-0.30^{\text {S*** }}$ & $-0.21^{5 *}$ & $-0.20^{5 *}$ & $-0.25^{5 * *}$ & -0.12 \\
\hline Diversion (Pos.Cop) & $-0.49^{P * * *}$ & $-0.48^{5 * * *}$ & $-0.35^{5 * * *}$ & $-0.32^{5 * * *}$ & $-0.36^{5 * * *}$ & $-0.26^{5 * *}$ \\
\hline Compensatory satisfaction (Pos.Cop) & $-0.30^{\mathrm{P} * * *}$ & $-0.32^{5 * * *}$ & $-0.18^{5 *}$ & $-0.19^{5 *}$ & $-0.22^{\mathrm{S} * *}$ & $-0.28^{5 * * *}$ \\
\hline Situation control (Pos.Cop) & $-0.30^{\mathrm{P} * * *}$ & $-0.17^{5 *}$ & $-0.20^{5 *}$ & $-0.22^{\mathrm{S} * *}$ & $-0.20^{5 *}$ & $-0.30^{5 * * *}$ \\
\hline Reaction control (Pos.Cop) & $-0.46^{\mathrm{P} * * *}$ & $-0.39^{\text {S**** }}$ & $-0.37^{\text {S*** }}$ & -0.39 s*** & $-0.44^{\text {S*** }}$ & $-0.33^{\text {S*** }}$ \\
\hline Positive self-instruction (Pos.Cop) & $-0.54^{\mathrm{P} * * *}$ & $-0.52^{\text {S*** }}$ & $-0.49^{5 * * *}$ & $-0.44^{5 * * *}$ & $-\left.0.5\right|^{5 * * *}$ & $-0.32^{5 * * *}$ \\
\hline Need for social support & -0.15 & -0.05 & -0.06 & -0.13 & -0.12 & -0.08 \\
\hline Active avoidance & $-0.24^{\mathrm{P} * *}$ & -0.13 & $-0.22^{5 * *}$ & $-0.21^{\mathrm{s*}}$ & $-0.19^{5 *}$ & -0.11 \\
\hline Escape tendency (Neg.Cop) & $0.36^{\mathrm{P} * * *}$ & $0.38^{5 * * *}$ & $0.32^{5 * * *}$ & $0.30^{5 * * *}$ & $0.38^{5 * * *}$ & $0.22^{5 * *}$ \\
\hline Rumination/perseveration (Neg.Cop) & $0.30^{\mathrm{P} * * * *}$ & $0.36^{5 * * *}$ & $0.34^{5 * * *}$ & $0.19^{5 *}$ & $0.35^{5 * * *}$ & 0.11 \\
\hline Resignation (Neg.Cop) & $0.5 I^{\mathrm{P} * * *}$ & $-0.50^{\text {s*** }}$ & $0.45^{5 * * *}$ & $0.38^{5 * * *}$ & $0.49^{5 * * *}$ & $0.34^{5 * * *}$ \\
\hline Self-accusation (Neg.Cop) & $0.42^{\mathrm{P} * * *}$ & $-0.53^{5 * * *}$ & $0.38^{5 * * *}$ & $0.295 * * *$ & $0.395 * * *$ & $0.27^{5 * *}$ \\
\hline
\end{tabular}

Notes: $* P<0.05 ; * * P<0.01 ; * * * P<0.001$.

Abbreviations: ISMI, Internalized Stigma of Mental Illness; ObjCGI, objective Clinical Global Impression; SubjCGI, subjective CGI; Pos.Cop, positive coping (SVF78); Neg. Cop, negative coping (SVF78); P, Pearson's r; S, Spearman's r; SVF, Strategie Zvládání Stresu (Stress Coping Style Questionnaire). 
Table 9 Multiple regression analysis with sum of Q-LES-Q or ISMI total as a dependent variable

\begin{tabular}{|c|c|c|c|c|c|c|}
\hline \multirow[t]{5}{*}{ Q-LES-Q } & Regressors & B & SE & $\beta$ & $t$ & Significance \\
\hline & Employment & -21.292 & 9.268 & -0.190 & -2.297 & 0.024 \\
\hline & ISMI total & $-1.87 \mid$ & 0.512 & -0.361 & -3.654 & 0.001 \\
\hline & Pos.Cop & 4.373 & 1.663 & 0.259 & 2.629 & 0.010 \\
\hline & \multicolumn{6}{|c|}{$F_{99}=I 7.19 I, P<0.00 I$; adjusted $R^{2}=0.329$} \\
\hline \multirow[t]{5}{*}{ ISMI total } & Regressors & B & $S E$ & $\beta$ & $t$ & Significance \\
\hline & SubjCGI & 2.667 & 0.925 & 0.264 & 2.883 & 0.005 \\
\hline & Pos.Cop & -0.750 & 0.339 & -0.224 & -2.212 & 0.030 \\
\hline & Neg.Cop & 0.475 & 0.250 & 0.178 & 1.903 & 0.060 \\
\hline & Q-LES-Q total & -0.049 & 0.017 & -0.260 & -2.833 & 0.006 \\
\hline
\end{tabular}

Abbreviations: Q-LES-Q, Quality of Life Enjoyment and Satisfaction Questionnaire; ISMI, Internalized Stigma of Mental IIIness; ObjCGI, objective Clinical Global Impression; SubjCGI, subjective CGI; Pos.Cop, positive coping; Neg.Cop, negative coping.

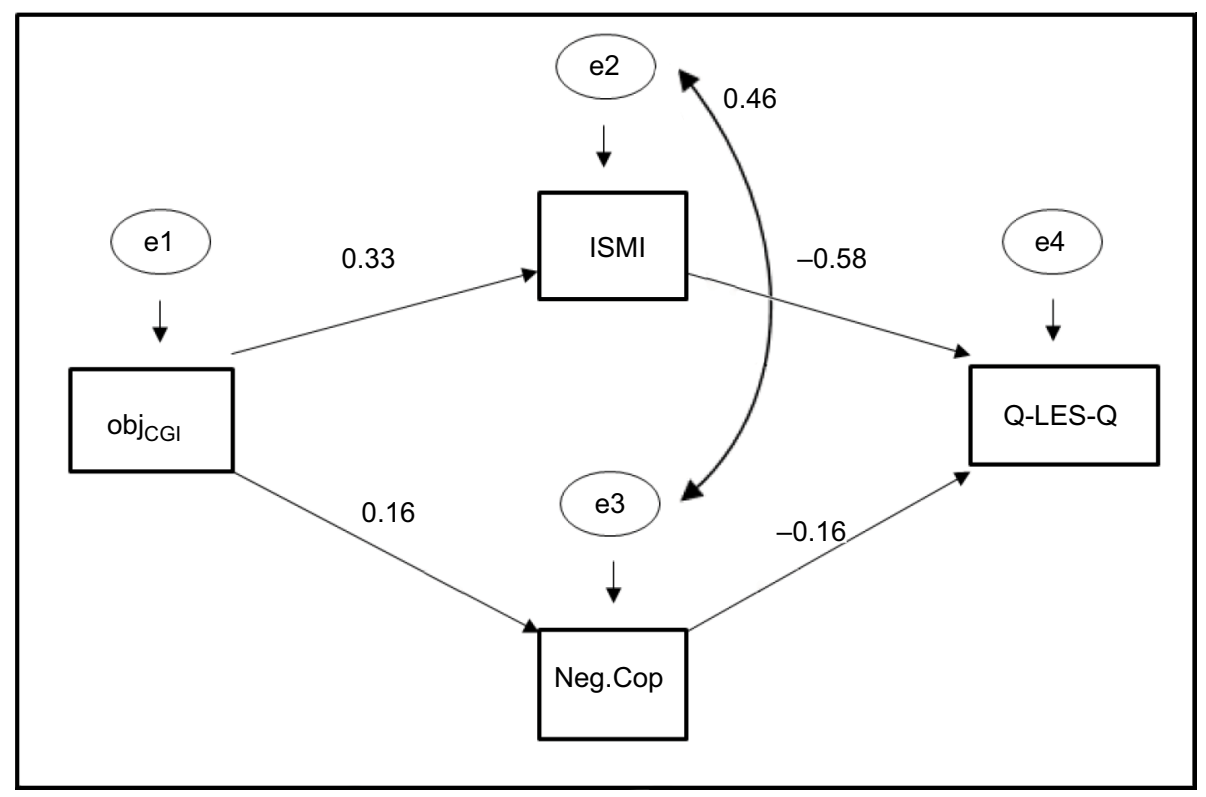

Figure I Mediation-analysis model.

Abbreviations: ISMI, Internalized Stigma of Mental Illness; ObjCGI, objective Clinical Global Impression; Q-LES-Q, Quality of Life Enjoyment and Satisfaction Questionnaire; Neg.Cop, negative coping.

\section{Mediation analysis}

Previous analyses showed that QoL (Q-LES-Q total) might be significantly influenced by Pos.Cop, Neg.Cop, self-stigma (ISMI total), and ObjCGI. Amos software was used for the analysis. The specific method was maximum likelihood, as it is a golden standard in structural equation modeling and robust against normality violations. ${ }^{38}$ Figure 1 shows standardized estimates of the pathways. The two paths linking ObjCGI with Q-LES-Q were significant. Fit indices also revealed a relatively appropriate model fit $\left(\chi_{1}^{2}=4.463\right.$ $[P=0.035]$, CFI 0.976, GFI 0.985, NFI 0.970, RMSEA 0.154). As such, levels of self-stigma and Neg.Cop strategies influenced QoL.

\section{Discussion}

This study evaluated relationships among demographic and clinical data, self-stigma, QoL, and coping strategies. The demographic characteristics of the sample were comparable to other outpatient investigations on NsD for age, age at onset, sex, and marital and occupational status. ${ }^{64-66}$ In the first analysis, relationships between self-stigma and QoL, coping strategies, and demographic data were evaluated. The findings showed that sex, partnership, education, and employment played no role in self-stigma. Identical results were found in other studies where no differences of self-stigma in connection with sex, ethnicity, education, or occupation were shown. ${ }^{36,37,67}$ Yen et al ${ }^{68}$ did not confirm associations between 
age, sex, and self-stigma either. On the other hand, Girma et $\mathrm{al}^{69}$ described higher self-stigma scores in women than in men. The level of self-stigma diminished with increasing levels of education. In the same way, participants who had a partner stigmatized themselves less than those without a partner. Also, the effect of education was important, as participants who had achieved higher education stigmatized themselves less. Marital status also influenced self-stigma. The differences in results could be attributed to different subpopulations of patients with various diagnostic and clinical conditions.

The findings showed that sex and partnership played no role in perceived QoL. However, QoL was different between patients with elementary education and patients with university education. Also, patients with a job displayed statistically significantly higher QoL than unemployed patients. These outcomes are similar to our results with patients with schizophrenia, where employed patients described higher QoL than unemployed patients. ${ }^{70}$

\section{Hypotheses}

\section{QOL will correlate negatively with self-stigma}

As in our previous studies in other patient groups, this hypothesis was confirmed. ${ }^{22,24,25,70}$ All Q-LES-Q domains negatively correlated with all ISMI domains, except school/study (probably caused by the small number of studying participants). It seems that patients who feel more socially excluded because of stigma experience lower QoL to a greater extent. The same applies to feelings of alienation from one another or society because of experiencing stigma. The findings are also in agreement with Vrbova et al, ${ }^{25}$ who also showed that selfstigma negatively predicted QoL in schizophrenic patients. Multiple-regression and mediation analyses confirm the results from correlations that self-stigma is one of the most powerful factors influencing QoL. Also, our previous studies showed that self-stigma correlated negatively with QoL in schizophrenia and depression. ${ }^{22,70}$

\section{QOL will correlate positively with positive coping and negatively with negative coping}

A hypothesis that QoL correlates positively with Pos.Cop was confirmed in correlation analysis and multiple regression. Pos.Cop strategies were significantly correlated with QoL. As Friedman et $\mathrm{al}^{53}$ pointed out, a positive approach to life may cause a promising effect on QoL in connection with Pos.Cop. However, the question is if patients who develop neurotic symptoms, such as anxiety, in reaction to stress can cope with stressful life events adaptively or not. Patients with social phobia use more Neg.Cop than others in that diagnostic category, so we can assume that Neg.Cop strategies, such as avoidant and escape tendencies and resignations used in this group of NsD due to ill-fated fear of contact with the outside world, are represented to a more considerably maladaptive degree. On the other hand, patients with panic disorder and agoraphobia may be evasive somewhat selectively in certain situations or focus specifically rather than in general. Therefore, they may have the lowest degree of negative management. Jacofsky et $\mathrm{al}^{49}$ pointed out that specific maladaptive coping strategies in different anxiety disorders affect the individual's capability to break the paired link between circumstances and symptoms of anxiety. Escape tendencies are frequently used by individuals with panic attacks and (social) phobia. Negative reinforcement (social phobia), safety behavior (panic and GAD), sensitization (GAD), and anxious avoidance (GAD, panic disorder, social phobia). Gattino et $\mathrm{al}^{71}$ posited that problem-focused approaches (positive reframing, active coping, using support) increase QoL in all its proportions, whereas emotion-focused coping (self-blame) reduces both psychological and physical QoL. Analogous outcomes were found in our previous studies on patients with schizophrenia and depression. ${ }^{22,43}$

On the other hand, mediation analysis indicated that QoL was mostly influenced by self-stigma and Neg.Cop strategies, which were both influenced by ObjCGI. It is interesting that according to the regression analysis, one of the main factors correlated with the Q-LES-Q were Pos.Cop strategies, but mediation analysis showed Neg.Cop strategies to be the main factor. In structural equation modeling, their interrelations are analyzed. Based on the theoretical background, several specific models explaining relationships between studied variables were selected. The significance of predictors of Q-LES-Q depends not only on isolated connections between each factor and the dependent variable but also on the structure of the model, ie, the associations among all these variables. As such, although Pos.Cop strategies were considered related to Q-LES-Q in multiple regression, they lost their impact in a model that tries to explain the relationships among the variables. Also, Neg.Cop strategies, which did not pass into the last step of the multiple regression (being pushed out by variables that showed a stronger isolated connection with the dependent variable) were meaningful in the supposed explanatory model. In this model, the factor of Neg.Cop strategies was a significant mediator. Therefore, it is not a strong dependent factor influencing Q-LES-Q, but significantly influences Q-LES-Q as a "middleman" between the severity of the disorder and Q-LES-Q. 


\section{QOL will correlate negatively with severity of disorder}

Subjectively and objectively evaluated severity of the disorder correlated negatively in most of the Q-LES-Q domains, except school/study. The mean age of the patients in the sample was around 48 years. We also confirmed this hypothesis in our previous study in schizophrenia, where QoL and severity of disorder were significantly related as well. ${ }^{70}$ Also, in another study, Q-LES-Q total and most Q-LES-Q domains correlated negatively with subjective and objective evaluation of the severity of the disorder in patients with depression. ${ }^{42}$

When subjectively and objectively evaluated severity of the disorder were put into multiple-regression analysis, they were removed by other more influential factors, such as self-stigma, employment, and Pos.Cop. However, in the mediation-analysis model, which considers interactions between factors, objective assessment of the severity of the disorder was a factor that impacted QoL indirectly through self-stigma and Neg.Cop strategies, as is visible in Figure 1.

\section{QOL will vary for different groups of neurotic} illnesses

The diagnostic subgroups differed significantly only in age and using Neg.Cop strategies, but not in other measured clinical or psychological variables. As such, such variables as severity of disorder, self-stigma, Pos.Cop, and QoL did not play a significant role in connection with the type of diagnosis. Norberg et $\mathrm{al}^{72}$ showed that patients diagnosed with anxiety disorders stated lower QoL than healthy controls. Reviews by Mogotsi et a $1^{73}$ and Mendlowicz and $\operatorname{Stein}^{5}$ about QoL in different anxiety disorders gave a practically identical portrait of anxiety disorders as disorders that noticeably decrease QoL and psychosocial functioning. A meta-analysis by Olatunji et a ${ }^{74}$ evaluated dissimilarities in QoL between individuals suffering from anxiety disorders and nonclinical controls. No anxiety-disorder diagnosis was linked with significantly poorer QoL than in any other diagnosis of anxiety disorder. On the contrary, Cramer et $\mathrm{al}^{10}$ investigated the influence of specific anxiety disorders on specific QoL indicators in a typical population. The outcomes indicated that panic disorder and social phobia within the past year, lifetime, and GAD within the past year had an independent influence on QoL when controlling for some sociodemographic variables, physical health, and other DSM-III-R Axis I psychiatric disorders. OCD and specific phobias had only an insignificant effect, and agoraphobia displayed no impact.
QOL will be significantly related to demographic factors, such as education, partnership, and employment

Employment is recognized as an essential factor in QoL. It is such a substantial factor that it passed the regression analysis as an independent factor. However, a connection between QoL and partnership was not found: patients with a partner had the same level of QoL as patients without a partner. Patients with elementary education had significantly lower QoL than university-graduated patients, but education was not a strong enough factor to pass the multiple regression analysis and did not show an effect in the mediation-analysis model.

QoL assessed by Q-LES-Q total showed a statistically significant difference between unemployed and employed patients. Employment was such a substantial factor that it passed the multiple-regression analysis as one of the three most powerful individual factors influencing QoL (with self-stigma and Pos.Cop). Having a job is quite an essential factor in QoL. Being employed was positively related to QoL in schizophrenia patients. ${ }^{75}$ However, the causal direction of this link is uncertain: employment did not show up as an essential factor in the mediation-analysis model. Our study did not show a correlation of QoL with partnership status of the patient, in opposition to many previous studies. ${ }^{75-77}$

\section{Self-stigma will correlate positively with severity of disorder}

Disorder severity, especially in connection with subjective perception of severity, was significantly associated with selfstigma in all domains. Subjective evaluation of the severity of the disorder also passed multiple-regression analysis as one of the most influential factors influencing self-stigma. Self-stigma is typically greater in participants with more severe symptoms of psychiatric disorder. ${ }^{22,24,35,36,54,67,70,78}$ It can also relate to the finding that patients with greater selfstigma tend to experience more serious signs of anxiety and depression..$^{58,79}$

\section{Self-stigma will correlate positively with negative coping and negatively with positive coping strategies} This hypothesis was confirmed in this study. Each of the coping strategies, whether positive or negative, except need for social support, which is considered a neutral strategy, was significantly related to self-stigma and its domains (except stigma resistance, in which most coping strategies were not associated). In the case of avoiding social contact within a self-stigma, positive self-instruction and resignation 
positively and negatively impacted the positive and negative effects of whether or not a particular individual was looking for a connection and could gain a positive experience with people and environment or whether the patient avoided social contact and hence could stigmatize themselves to a greater extent. Rüsch et $\mathrm{l}^{80}$ showed that persons who stigmatize themselves have a tendency to experience social anxiety. Social anxiety produces and is strengthened by safety and avoidant behavior. Persons with internalized stigma have a tendency to isolate themselves from society ${ }^{81}$ In our previous investigation of schizophrenia-spectrum-disorder patients, we also found significant associations between coping strategies and self-stigma. The capacity to use Pos.Cop is linked to decreasing self-stigma, and using Neg.Cop strategies mainly increases the self-stigma. ${ }^{70} \mathrm{We}$ cannot answer questions about causality in this cross-sectional study. Positive self-instruction and resignation are also essential strategies relating to alienation and stereotype agreement.

The regression analysis indicated that the most important aspects associated with QoL were Pos.Cop strategies, employment, and total self-stigma score. Other factors were not strong enough to pass the regression analysis. According to many studies, these factors are important in QoL..$^{5,10,22,53,72-75}$ It seems that there could exist a significant interaction between having a job as an essential part of QoL and good life feeling, which can be associated with positive management of life events and reduced self-stigma because of a positive perception of one's self. A cross-sectional study cannot answer questions on interactions and causality among these variables.

\section{Limitations}

The study had several shortcomings. The information was derived primarily from self-assessment. The use of such methods is limited by the ability of self-reflection of participants and willingness to be open with their information. Another limitation was the broad range of the NsD and comorbidities that the probands experienced. We did not assess the level of depression in this cross-sectional study, because "current depressive episode" was in the exclusion criteria. Serious somatic illness was also in the exclusion criteria. The patients were also treated with different medications, but the exact doses of the drug used were not monitored in this study.

\section{Conclusion}

Self-stigma, QoL, and coping strategies were revealed as important factors for people with NsD. The study confirmed links between self-stigma, QoL, severity of disorder, and coping strategies of outpatients with NsD. Self-stigma was not affected so much by the particular diagnosis. However, participants with higher severity of disorder, higher self-stigma, and Neg.Cop described a lower QoL. Future investigations should focus on paths of self-stigma and the impact of coping strategies on QoL in longitudinal studies of NsD.

Suggestions for practice include conveying the message to clinical workers that self-stigma, coping strategies, and QoL are interconnected. Speaking with patients about their understanding of being a psychiatric patient and being "cured", understanding their fears of changes in life roles and their self-stigma directly, and encouraging them to change this opinion of themselves can be reasonably central for patients and their recovery.

\section{Acknowledgments}

The authors are grateful for the willing cooperation of Dr Jiří Rozkoš from Prostějov, Dr Jan Flídr from Kralupy nad Vltavou, Dr Jana Matějková from Prague, Dr Simona Papežová from Prague, Dr Pavel Tautermann from Prague, Dr Markéta Zemanová from Havlíčkův Brod, Dr Markéta Dobrá from Hrabyně, Dr Zuzana Kozáková from Trutnov (RIAPS association), Dr Tibor Miklóš from Prague, Dr Michaela Zapletalová from Chomutov, Dr Jana Novosadová from Blansko, Dr Jiří Trska from Týn nad Vltavou, Dr Zdeněk Holoubek from Nymburk, Dr Iva Ondráčková from Prague, Dr Andrea Bryčková from Hořice, Dr Jindřiška Masnerová from Beroun, Dr Petr Pastucha from Prostějov, and Dr Hana Lemanová from Brno.

\section{Disclosure}

The authors report no conflicts of interest in this work.

\section{References}

1. International Classification of Disorders (1996) 10th revision. Mental and behavioural disorders: research diagnostic criteria. Prague: Prague Psychiatric Centre; 1996:179.

2. Bandelow B, Michaelis S. Epidemiology of anxiety disorders in the 21st century. Dialogues Clin Neurosci. 2015;17(3):327-335.

3. ÚŽIS ČR. Activity in branch of psychiatry. Institute of Health Information and Statistics Czech Republic. 2005; 27. Available from: https:// www.uzis.cz/system/files/zdrroccz2005.pdf. Accessed January 30, 2019.

4. Ormel J, Jeronimus BF, Kotov R, et al. Neuroticism and common mental disorders: meaning and utility of a complex relationship. Clin Psychol Rev. 20131;33(5):686-697.

5. Mendlowicz MV, Stein MB. Quality of life in individuals with anxiety disorders. Am J Psychiatry. 2000;157(5):669-682.

6. Ansseau M, Dierick M, Buntinkx F, et al. High prevalence of mental disorders in primary care. J Affect Disord. 2004;78(1):49-55.

7. Stevanovic D. Impact of emotional and behavioral symptoms on quality of life in children and adolescents. Qual Life Res. 2013;22(2):333-337. 
8. Raphael J, Paul K. Psychological well-being, and anxiety among adolescents analysis along wellness: illness continuum. Int J Innov Res Dev. 2014;3:395-401.

9. Raknes S, Pallesen S, Himle JA. Quality of life in anxious adolescents. Child Adolesc Psychiatry Ment Health. 2017;112017(1):33.

10. CramerV, Torgersen S, Kringlen E. Quality of life and anxiety disorders: a population study. J Nerv Ment Dis. 2005;193(3):196-202.

11. Weidle B, Jozefiak T, Ivarsson T, Thomsen PH. Quality of life in children with OCD with and without comorbidity. Health Qual Life Outcomes. 2014;12(1):1-12.

12. Hoff AL, Kendall PC, Langley A, et al. Developmental differences in functioning in youth with social phobia. J Clin Child Adolesc Psychol. 2017;46(5):686-694.

13. Loh DA, Moy FM, Zaharan NL, Mohamed Z. Disparities in healthrelated quality of life among healthy adolescents in a developing country - the impact of gender, ethnicity, socio-economic status and weight status. Child Care Health Dev. 2015;41(6):1216-1226.

14. Holly LE, Little M, Pina AA, Caterino LC. Assessment of anxiety symptoms in school children: a cross-sex and ethnic examination. $J$ Abnorm Child Psychol. 2015;43(2):297-309.

15. Pantol G, Schneier FR. Quality of life in anxiety disorders. Qual Life Ment Disord. 1997:149-164.

16. Development of the World Health Organization WHOQOL-BREF quality of life assessment. The WHOQOL group. Psychol Med. 1998;28:551-558.

17. World Health Organization Quality of Life assessment (WHOQOL). Position paper from the World Health Organization. Soc Sci Med. 1995;41(10):1403-149.

18. Taghipour H, Moharamzad Y, Mafi AR, et al. Quality of life among Veterans with war-related unilateral lower extremity amputation: a long-term survey in a prosthesis center in Iran. J Orthop Trauma. 2009;23(7):525-530.

19. Rahimi A, Mousavi B, Soroush M, Masumi M, Montazeri A. Pain and health-related quality of life in war veterans with bilateral lower limb amputations. Trauma Mon. 2012;17(2):282-286.

20. Katschnig H. Quality of life in mental disorders: challenges for research and clinical practice. World Psychiatry. 2006;5(3):139-145.

21. Powdthavee N, Lekfuangfu WN, Wooden M. What's the good of education on our overall quality of life? A simultaneous equation model of education and life satisfaction for Australia. J Behav Exp Econ. 2015;54:10-21.

22. Holubova M, Prasko J, Matousek S, et al. Comparison of self-stigma and quality of life in patients with depressive disorders and schizophrenia spectrum disorders - a cross-sectional study. Neuropsychiatr Dis Treat. 2016;12:3021-3030.

23. Picco L, Pang S, Lau YW, et al. Internalized stigma among psychiatric outpatients: associations with quality of life, functioning, hope and self-esteem. Psychiatry Res. 2016;246:500-506.

24. Vrbova K, Prasko J, Ociskova M, et al. Quality of life, self-stigma, and hope in schizophrenia spectrum disorders: a cross-sectional study. Neuropsychiatr Dis Treat. 2017;13:567-576.

25. Vrbova K, Prasko J, Ociskova M, Holubova M. Comorbidity of schizophrenia and social phobia - impact on quality of life, hope, and personality traits: a cross sectional study. Neuropsychiatr Dis Treat. 2017;13: 2073-2083.

26. Brook OH, van Hout HP, Nieuwenhuysea H, de Haan M. Effects of coaching by community pharmacists on psychological symptoms of antidepressant users; a randomised controlled trial. Eur Neuropsychopharmacol. 2003;13(5):347-354.

27. Corrigan PW, Rafacz J, Rüsch N. Examining a progressive model of self-stigma and its impact on people with serious mental illness. Psychiatry Res. 2011;189(3):339-343.

28. Parcesepe AM, Cabassa LJ. Public stigma of mental illness in the United States: a systematic literature review. Adm Policy Ment Health. 2013;40(5):384-399.

29. Overton SL, Medina SL. The stigma of mental illness. J Couns Dev. 2008;86(2):143-151.
30. Sirey JA, Bruce ML, Alexopoulos GS, et al. Perceived stigma as a predictor of treatment discontinuation in young and older outpatients with depression. Am J Psychiatry. 2001;158(3):479-481.

31. Tsang HW, Fung KM, Chung RC. Self-stigma and stages of change as predictors of treatment adherence of individuals with schizophrenia. Psychiatry Res. 2010;180(1):10-15.

32. Padurariu M, Ciobica A, Persson C, Stefanescu C. Self-stigma in psychiatry: ethical and bio-psychosocial perspectives. Rom J Bioeth. 2011;9(1):76-82.

33. Ustündağ MF, Kesebir S. Internalized stigmatization in bipolar patients: relationship with clinical properties, quality of life and treatment compliance. Turk Psikiyatri Derg. 2013;24(4):231-239.

34. Ociskova M, Prasko J, Kamaradova D, Grambal A, Latalova K, Sigmundova Z. Relationship between internalized stigma and treatment efficacy in mixed neurotic spectrum and depressive disorders. Neuro Endocrinol Lett. 2014; 35(8):711-717.

35. Cinculova A, Prasko J, Kamaradova D, et al. Self-stigma and discontinuation of pharmacotherapy in patients with anxiety disorders - crosssectional study. Neuro Endocrinol Lett. 2017;38(6):429-426.

36. Kamaradova D, Latalova K, Prasko J, et al. Connection between selfstigma, adherence to treatment, and discontinuation of medication. Patient Prefer Adherence. 2016;22(10):1289-1298.

37. Camp DL, Finlay WML, Lyons E. Is low self-esteem an inevitable consequence of stigma? An example from women with chronic mental health problems. Soc Sci Med. 2002;55(5):823-834.

38. Finney SJ, DiStefano C. Non-normal and categorical data in structural equation modeling. In: Hancock GR, Mueller RD, editors. Structural Equation Modeling: A Second Course. Greenwich: Information Age Publishing; 2008; 269-314.

39. Barney LJ, Griffiths KM, Christensen H, Jorm AF. Exploring the nature of stigmatising beliefs about depression and help-seeking: implications for reducing stigma. BMC Public Health. 2009;9(1):61.

40. Link BG, Phelan JC, Stigma C. Conceptualizing stigma. Annu Rev Sociol. 2001;27(1):363-385.

41. Alonso J, Buron A, Bruffaerts R, et al. World Mental Health Consortium. Association of perceived stigma and mood and anxiety disorders: results from the world mental health surveys. Acta Psychiatr Scand. 2008;118(4):305-314.

42. Holubova M, Prasko J, Ociskova M, et al. Quality of life and coping strategies of outpatients with a depressive disorder in maintenance therapy - A cross-sectional study. Neuropsychiatr Dis Treat. 2017;14:73-82.

43. Holubova M, Prasko J, Hruby R, et al. Coping strategies and quality of life in schizophrenia: cross-sectional study. Neuropsychiatr Dis Treat. 2015;11:3041-3048.

44. Marquez-Arrico JE, Benaiges I, Adan A. Strategies to cope with treatment in substance use disorder male patients with and without schizophrenia. Psychiatry Res. 2015;228(3):752-759.

45. Folkman S, Moskowitz JT. Coping: pitfalls and promise. Annu Rev Psychol. 2004;55(1):745-774.

46. Skinner EA, Edge K, Altman J, Sherwood H. Searching for the structure of coping: a review and critique of category systems for classifying ways of coping. Psychol Bull. 2003;129(2):216-269.

47. Janke W, Erdmann G. SVF 78: Eine Kurzform des Stressverarbeitungsfragebogen SVF 120 [A short form of stress management questionnaire SVF 120]. GöttingenGerman: Hogrefe Verlag für Psychologie; 2002.

48. Aldwin CM. Stress, Coping, \& Development: An Integrative Perspective. 2nd ed. New York, NY: Guildford Press; 2007.

49. Jacofsky MD, Santos MT, Neziroglu F. The Maintenance Of Anxiety Disorders: Maladaptive Coping Strategies. MentalHelp.net [online]. San Diego: Centersite, 2009 [cit. 2018-07-02]. Available from: https://www.mentalhelp.net/articles/the-maintenance-of-anxietydisorders-maladaptive-coping-strategies/. Accessed January 15, 2019.

50. Bch K, Arnold R, Rodriguez-Rubio B. Mediating effects of coping in the link between spirituality and psychological distress in a culturally diverse undergraduate sample. Ment Health Relig Cult. 2014;17(2):173-184.

51. Folkman S. Personal control and stress and coping processes: a theoretical analysis. J Pers Soc Psychol. 1984;46(4):839-852. 
52. Scheier MF, Matthews KA, Owens JF, et al. Dispositional optimism and recovery from coronary artery bypass surgery: the beneficial effects on physical and psychological well-being. J Pers Soc Psychol. 1989;57(6):1024-1040.

53. Friedman LC, Kalidas M, Elledge R, Optimism ER, et al. Optimism, social support and psychosocial functioning among women with breast cancer. Psychooncology. 2006;15(7):595-603.

54. Ociskova M, Prasko J, Kamaradova D, Grambal A, Sigmundova Z. Individual correlates of self-stigma in patients with anxiety disorders with and without comorbidities. Neuropsychiatr Dis Treat. 2015;11:1767-1779.

55. Ritsner M, Kurs R, Gibel A, Ratner Y, Endicott J. Validity of an abbreviated Quality of Life Enjoyment and Satisfaction Questionnaire (Q-LES-Q-18) for schizophrenia, schizoaffective, and mood disorder patients. Qual Life Res. 2005;14(7):1693-1703.

56. Müllerova $H$. Transcultural transmission and validation of the quality life questionnaire Q-LES-Q. (in Czech: Mezikulturní přenos a validace dotazníku kvality života Q-LES-Q.). Psychiatrie. 2001;5:80-87.

57. Ritsher JB, Otilingam PG, Grajales M. Internalized stigma of mental illness: psychometric properties of a new measure. Psychiatry Res. 2003;121(1):31-49.

58. Boyd JE, Adler EP, Otilingam PG, Peters T. Internalized stigma of mental illness (ISMI) scale: a multinational review. Compr Psychiatry. 2014;55(1):221-231.

59. Ocisková M, Praško J, Kamarádová D, et al. Self-stigma in psychiatric patients-standardization of the ISMI scale. Neuro Endocrinol Lett. 2014;35(7):624-632

60. Guy W, editor. ECDEU assessment manual for psychopharmacology. Rockville:U.S. DHEW; 1976.

61. Zaider TI, Heimberg RG, Fresco DM, Schneier FR, Liebowitz MR. Evaluation of the clinical global impression scale among individuals with social anxiety disorder. Psychol Med. 2003;33(4):611-622.

62. Svancara J. Coping Strategies. Testcentrum, Prague; 2003.

63. EMEA; 2002. Available from: http://www.ema.europa.eu/docs/en_GB/ document_library/Scientific_guideline/2009/09/WC500002874.pdf. Accessed March 20, 2009.

64. Michael T, Zetsche U, Margraf J. Epidemiology of anxiety disorders. Psychiatry. 2007;6(4):136-142.

65. Prasko J, Kamaradova D, Cakirpaloglu S, et al. Predicting the therapeutic response to intensive psychotherapeutic programs in patients with neurotic spectrum disorders. Activitas Nervosa Super Rediviva. 2015;57(1-2):30-39.

66. Ociskova M, Prasko J, Latalova K, Kamaradova D, Grambal A. Psychological factors and treatment effectiveness in resistant anxiety disorders in highly comorbid inpatients. Neuropsychiatr Dis Treat. 2016;12:1539-1551.
67. Livingston JD, Boyd JE. Correlates and consequences of internalized stigma for people living with mental illness: a systematic review and meta-analysis. Soc Sci Med. 2010;71(12):2150-2161.

68. Yen CF, Chen CC, Lee Y, Tang TC, Yen JY, Ko CH. Self-stigma and its correlates among outpatients with depressive disorders. Psychiatr Serv. 2005;56(5):599-601.

69. Girma E, Tesfaye M, Froeschl G, Möller-Leimkühler AM, Dehning S, Müller N. Facility based cross-sectional study of self stigma among people with mental illness: towards patient empowerment approach. Int J Ment Health Syst. 2013;7(1):21.

70. Holubova M, Prasko J, Hruby R, et al. Coping strategies and self-stigma in patients with schizophrenia-spectrum disorders. Patient Prefer Adherence. 2016;10:1151-1158.

71. Gattino S, Rollero C, De Piccoli N. Applied research quality life: the influence of coping strategies on quality of life from a gender perspective. $2015 ; 10: 689$.

72. Norberg MM, Diefenbach GJ, Tolin DF. Quality of life and anxiety and depressive disorder comorbidity. J Anxiety Disord. 2008;22(8): 1516-1522.

73. Mogotsi M, Kaminer D, Stein DJ. Quality of life in the anxiety disorders. Harv Rev Psychiatry. 2000;8(6):273-282.

74. Olatunji BO, Cisler JM, Tolin DF. Quality of life in the anxiety disorders: a meta-analytic review. Clin Psychol Rev. 2007;27(5):572-581.

75. Bouwmans C, De Sonneville C, Mulder CL, Hakkaart-van Roijen L. Employment and the associated impact on quality of life in people diagnosed with schizophrenia. Neuropsychiatr Dis Treat. 2015; 11: 2125-2142.

76. Üçok A, Gorwood P, Karaday G; EGOFORS. Employment and its relationship with functionality and quality of life in patients with schizophrenia: EGOFORS Study. Eur Psychiatry. 2012;27(6): 422-425.

77. Ra Y-A, Kim WH. Impact of employment and age on quality of life of individuals with disabilities: a multilevel analysis. Rehabil Couns Bull. 2016;59(2):112-120.

78. Latalova K, Prasko J, Kamaradova D, et al. Self-stigma and suicidality in patients with neurotic spectrum disorder - a cross sectional study. Neuro Endocrinol Lett. 2014;35(6):474-480.

79. Ritsher JB, Phelan JC. Internalized stigma predicts erosion of morale among psychiatric outpatients. Psychiatry Res. 2004;129(3): 257-265.

80. Rüsch N, Corrigan PW, Powell K, et al. A stress-coping model of mental illness stigma: II. emotional stress responses, coping behavior and outcome. Schizophr Res. 2009;110(1-3):65-71.

81. Vauth R, Kleim B, Wirtz M, Corrigan PW. Self-efficacy and empowerment as outcomes of self-stigmatizing and coping in schizophrenia. Psychiatry Res. 2007;150(1):71-80.
Psychology Research and Behavior Management

\section{Publish your work in this journal}

Psychology Research and Behavior Management is an international, peerreviewed, open access journal focusing on the science of psychology and its application in behavior management to develop improved outcomes in the clinical, educational, sports and business arenas. Specific topics covered in the journal include: Neuroscience, memory and decision making; Behavior modification and management; Clinical applications; Business and sports performance management; Social and developmental studies; Animal studies. The manuscript management system is completely online and includes a very quick and fair peer-review system, which is all easy to use. Visit http://www. dovepress.com/testimonials.php to read real quotes from published authors. 